\title{
MANAGEMENT OF AMPUTEES
}

\section{A TEAM APPROACH}

\author{
by \\ BeRNICE KegeL, B.Sc., Physio. (Rand) R.P.T. \\ Staff Physical Therapist Universify of Chicago Hospital and Clinics
}

The need for comprehensive prosthetic services is ever increasing. Unfortunately in many institutions an interdisciplinary approach is not utilized. Physicians, Physiotherapists, Occupational Therapists, Prosthetists, Social Workers and nursing staff operate as individuals with no communication other than prescriptions and progress reports. This is not always in the best interest of the patient and therefore the concept of an Amputation Clinic is recommended as an alternative.

A comprehensive clinic has been in operation at the University of Chicago for the past three years, and could probably be initiated at any hospital, involving minimal effort or expense. No major renovations are necessary. The Physiotherapy Gymnasium is an appropriate place to hold the clinic, as parallel bars and walking aids are readily available. The cost of establishing and operating this clinic is also low. No new employees need be hired to implement the programme, merely reorganization of the present staff who continue to maintain their general case load.

The Amputation Clinic at this facility is held for $2 \frac{1}{2}$ hours once a month. Approximately 10 patients are seen every month, thus each patient receives 15 minutes of individual attention. Obviously, frequency of the clinic would depend on patient load in each particular hospital.

The clinic is attended by the Orthopaedic Surgeon (or Cardiovascular Surgeon where applicable), the Prosthetist (a private company in this incident), the Physiotherapist, Occupational Therapist and Social Worker. A file on each patient containing their history, operative and progress reports relevant to the case is kept in the Physiotherapy department. The Physiotherapist co-ordinating the clinic is called upon to meet and evaluate each patient before amputation (wherever applicable). The Physician and Physiotherapist work together in the early post-operative hases of rehabilitation. Physician, Prosthetist and Physiotherapist are present during rigid dressing changes in order to examine the condition of the residual limb and to plan a progressive weight bearing regime for the patient. It is the responsibility of the Physiotherapist to assure that the patient is scheduled to be seen in clinic as soon as the "shaping" of the residual limb has been completed. At the clinic the Physiotherapist presents the patient, offering a brief synopsis of his history and post-operative course, as well as the reason for the present visit. A group discussion is then undertaken with each member of the team participating in the discussion. A decision is made as to whether the patient is indeed a prosthetic candidate, and if so, which prosthetic device is indicated for that particular patient. The most appropriate prescription is made with the Physician using medical discretion, the Prosthetist having input into what is prosthetically available, the Physiotherapist explaining the patient's physical abilities and limitations and the patient expressing his own desires and expectations. A standardized prosthetic prescription form (see figure 1) is filled out by the Physician in duplicate. One copy is kept by the Prosthetist, and one copy kept in the patient's file. If this procedure is used, unnezessary time consuming telephone calls or letters are eliminated. The Social Worker then discusses whatever social and financial arrangements that are appropri- ate. Appointments are then made for the patient to go to the prosthetic company for casting and fitting. Using this protocol the prosthesis is complete within 1 to 6 weeks depending upon work load and complexity of the device.

As soon as the prosthesis has been fabricated it is sent to the Physiotherapy department - never ever directly to the patient, or via the patient. Gait training is then initiated, preferably daily, but usually three times per week. Occasionally, if transportation or other difficulties arise, training may be performed on an inpatient basis. The Physiotherapist does both an initial and final checkout of the prosthesis (a standardized form is used as shown in figure 2). Any minor changes or corrections necessary are discussed with the Prosthetist and completed before further training is commenced, so as to prevent poor gait patterns due to an inadequately aligned prosthesis.

Period of training required is highly variable. Sixty eight patients were trained at the University of Chicago in the past three years. Training time varied from 2 weeks to 7 months with a mean of 1,9 months. Two months on the average was required to prosthetically train a below knee amputee, and three months for an above knee amputee. This information is based on a frequency of treatment averaging at twice a week.

When the Physiotherapist believes the patient has obtained optimal physical benefits from training she refers him back to the amputation clinic. A group checkout is then done by Physician, Prosthetist and Physiotherapist or Occupational Therapist. The patient is evaluated for comfort, fit, alignment, function and cosmesis. He is asked to demonstrate his skill in handling and using the prosthesis. If both the team and the patient are satisfied, the patient is temporarily discharged.

This is not the end. Residual limbs change, prostheses wear out, and vascular changes occur in other extremities. Regular checkups have to be offered to these patients. The jrequency of rechecks will depend on age, reason for amputation, activity level and reliability of the patient. In this clinic, vascular patients are seen at three monthly intervals. Juvenile amputees are also scheduled extremely frequently so as to observe carefully for growth and bony overgrowth, a common problem in growing stumps. Patients who have had the amputation for neoplasm or trauma seldom need to be seen more than once a year in the amputation clinic per se. Whatever the problem, each patient should be given a card with the telephone numbers of all the appropriate professional personnel so that he might consult them at any time deemed necessary. The Social Worker or Vocational Counselor may continue to have contact with the patient to assure smooth transition to his new life situation or adaptation to a changing labour market.

Kerstein and associates (1974) reported that a medium of $5 \frac{1}{2}$ months transpired between time of amputation and final discharge from Physiotherapy. This is a long time, especially when one considers that the majority of patients are in the older age group anyway. Continued emphasis and effort should be afforded aiming at reducing the length of time involved. A great deal of time is usually lost due to lack of organization on the part of the staff involved rather than medical problems inherent in the patient. This time 
period could be substantially reduced by having an efficient and frequently run clinic catering to the needs of these patients.

By keeping accurate records, and following patients up on a regular basis, one is afforded an excellent opportunity for clinical and statistical research, something so sorely lacking in many spheres of rehabilitation.

Last but not least the clinic situation offers an ideal situation for interdisciplinary teaching. It offers a learning experience for medical, physiotherapy, occupational therapy and social work students. Both therapist and physician are offered the opportunity of learning as much about prosthetics as they desire, a topic usually skimmed over at the undergraduate level.

Figure 1: Below Knee Amputee Prosthetic Prescription.

Name:
Sex:
Height:

Date:

Construction:

Exoskeletal:

Endoskeletal Modular:

Socket:

P.T.B. with Soft Leather Liner.

P.T.B. Hard Socket.

P.T.B. Hard with Soft End.

P.T.B. Air Cushion.

Plug Fit.

P.T.S.

\section{Socket Material: \\ Plastic. \\ Wood.}

Suspension :

Cuff.

Supracondylar, suprapatellar.

Supracondylar with removable wedge.

Thigh lacer.

Waist Belt.

Ankle/foot:
Sach.
Single axis.
Double Axis.

Heel:

Soft.

Medium.

Hard.

Special Alignment Problems:

Stump Socks :

Number.

Ply.

Wool.

Cotton.

Other.

Remarks :

Prosthetic Facility.

M.D.

Key:

P.T.B. = Patella Tendon Bearing.

P.T.S. = Patella Tendon Supracondylar.

Sach. $=$ Solid Ankle Cushion Heel.
Figure 2: Checkout of Below Knee Prosthesis.

Adapted from forms used at Northwestern University, Chicago and University of California, Los Angeles.

Name of Patient:

Date of Initial Checkout:

Date of Final Checkout:

1. Is the prosthesis as prescribed? Are any changes justified.

Check With Patient Standing In Parallel Bars.

2. Is the pelvis level when the amputee bears weight equally on both feet?

3. Is the patient comfortable?

4. Is the socket comfortable in the distal anterior area of the tibia? (Pressure for anterior knee stability is exerted here but it should not be uncomfortable).

5. Is the socket comfortable over the end of the fibula?

6 . Is the knee stable on weight bearing?

7. Does the sole of the shoe maintain even contact with the floor?

8. Are tissue rolls around the trim line of the socket and the cuff suspension minimal?

9. Does the anterior trim line extend to the middle of the patella? (unless P.T.S.).

10. Does the insert extend above the trim line of the socket?

11. Is there gapping at the brim of the socket?

12. Is the stump in contact with the distal end of the socket? (Test with a ball of clay or powder).

13. Does the suspension system maintain it's proper position as the foot is elevated off the floor?

Check With the Patient Sitting.

14. Is the amputee comfortable while sitting with the sole of the shoe flat on the floor?

15. Is there adequate flaring of the posterior trim line to accommodate the hamstring tendons?

16. With the sole of the shoe flat on the floor is the anterior gap of the socket minimal? (Less than $\frac{3}{4}$ inch is acceptable).

17. Can the patient flex knee to 90 degrees?

18. Is the residual limb forced out of the socket excessively?

19. Are the colour and contour of the prosthesis similar to the sound leg?

Check With the Patient Walking.

20. Is gait satisfactory?

If unsatisfactory, which of the following deviations exist?

(a) Heel Strike:

Instability of the knee.

Knee extension.

Exaggerated knee flexion.

(b) Mid-Stance:

Lateral bending of trunk.

Wide base.

Shoe not flat on floor.

Excessive lateral pressure over fibula head.

Unequal weight bearing time.

Lateral gapping at socket brin.

Medial gapping at socket brim.

(c) Push-Off:

Pelvic rise or "hill climbing".

Pelvic drop-off.

(d) Swing Phase:

Excessive piston action.

Excessive knee flexion.

Vaulting on sound side.

Circumduction.

Erratic line of foot swing.

Unequal step length. 
Advanced Activities.

23. Does the amputee ascend and descend stairs safely? (with and without rails).

24. Can the amputee walk on uneven ground?

26. Are there sock impressions over the entire stump?

27. Is the skin free of abrasions or blisters?

28. Are the weight bearing areas on the skin of the residual limb free of pain and discomfort?

Recommendations.
21. Does the amputee ascend inclines satisfactorily?

22. Does the amputee descend inclines satisfactorily?

25. Can the amputee get in and out of a car?

Check with the Prosthesis off.

\section{Post Registration} Courses

\author{
POST GRADUATE COURSE
}

UNIVERSITY OF CAPE TOWN, DEPARTMENT OF PHYSIOTHERAPY

AND

THE CENTRE OF EXTRA-MURAL STUDIES

\author{
"Modern Approaches to the \\ Physiotherapeutic Treatment \\ in the Latter Stages of \\ Brain Injury"
}

3. Chapman, C. E., Palmer, H. F., Bell, D. M., Follow up study on a Group of Older Patients. Journal of the American Medical Association, Vol. 170: 1396-1402, July 18, 1959.

4. Kerstein, M. D., Zimmer, H., Dugdale, F. E., Lerner, E., Amputation of the Lower Extremity, a Study of 194 cases. Arch, Phys. Med. Rehabil. Vol. 55, October, 1974.

5. Stern, M., Payton, O. D., Establishing a Prosthetic Clinic in an Acute Care Facility. Hospital Topics, January, 1970.

\section{Books Received}

PHYSIOTHERAPY IN PAEDIATRIC PRACTICE, by Scrutton \& Gilbertson (1975).

Price: R20,55 plus 25c delivery.

\section{POSTURAL VARIATIONS IN CHILDHOOD,}

by C. Asher (1975).

Price: R 12,75 plus $15 \mathrm{c}$ delivery.

Both the above books are included in the Postgraduate Paediatric Series and are obtainable from Butterworth \& Co. (S.A.) (Pty) Ltd., P.O. Box 792, Durban 4000.
Guest Lecturers:

MRS. B. GOFF

Exponent of Rood Techniques

PROFESSOR J. C. DE VILLIERS

Neuro Surgeon

Course maximum: 24 members.

Registration fee (full course): R30,00.

Individual Lectures available to doctors and other interested persons: $\mathrm{R} 4,00$ per session.

U.C.T. Accommodation available:

R10,00 per day. 